\title{
Research on the Application of Geometric Graphics Creative Elements in Graphic Advertisement Design
}

\author{
Yiyi Yang \\ Design Art \& Fashion Institute of Xiamen University of Technology, Xiamen Fujian, 361024, China
}

Keywords: Graphic design, Geometric graphics, Creative elements, Application.

\begin{abstract}
With the development of culture, China's advertising industry has shown a trend of high-speed operation, whether it is the number or type are increasing, in the graphic advertising design application of different geometric graphics creativity, has a very important artistic value, Element handling and control value while ensuring that print ads can play their own advantages. This paper analyzes the present situation of application of graphic creative elements in graphic advertising design, and explains in detail the significance of the application of graphic creative elements in graphic advertising design, and focuses on the practical application measures. It aims to provide relevant designers with Valuable advice.
\end{abstract}

\section{Introduction}

It is in the context of the continuous development of digital technology, print ads and multimedia advertising has become a normal form of advertising, the traditional print advertising is also under the influence of multimedia technology to achieve a diversified development of the design language, whether it is dynamic elements or geometry Creative elements, are presented with efficient design efficiency, to ensure that aesthetic design rules and control requirements meet the standards.

\section{Application Status of Geometric Graphics Creative Elements in Graphic Advertisement Design}

The application of geometric graphic elements in graphic advertising design is an inevitable choice to adapt to the development trend of the times. Geometric graphic elements occupy a very important position in graphic advertising with its visual form of beauty, especially in the actual design and construction process, The designer with creative elements to improve the beauty of the screen, the formation of a variety of visual communication, in the process of combining the creative elements of the actual value of a very important significance. The geometric graphics creative elements as the main design language can improve the overall picture of beauty at the same time, establish a sound system of geometric graphics creative model.

Geometric graphic elements of creativity driven by the elements of the plane have become a multimedia advertising design language, to the virtual community. In the actual design structure, the geometric graphic elements are not only used in the graphic design of the point, line, surface and other basic modeling elements, including the random combination of graphics and geometric graphics of the effective splicing, to achieve the overall creative structure of the update and Development, and its color, texture and other visual elements are more diversified. It is based on this, in the print ads, the application of geometric graphics creative elements can achieve visual communication. Print ads incorporate geometric graphic elements into the design language to create more innovative applications that ensure that the overall design structure is optimized. In the graphic design application of geometric creative graphics, so that in many media advertising highlights their own characteristics. From the elements in the traditional advertising actively learn and absorb, can effectively show their own unique [1]. 


\section{Significance of Applying Geometric Graphic Elements in Graphic Advertisement Design}

\section{Application of Geometric Graphic Elements in Graphic Advertisement Design}

Application of geometric graphics in the design of graphic advertising on the basis of the original, to enhance the performance of the overall picture of the application effect, to achieve the use of the law of integration and historical aesthetic and development, can improve the attractiveness of the screen, breaking the traditional single design Structure and visual space experience, but also makes the picture of the dynamic and emotional attitude more realistic, but also directly affect the mood of the viewer. In the process of applying the creative elements of geometric shapes, different transform combinations are established, which can enhance the visual appeal while improving the visual effect. For example, at the 2012 London Olympics, the use of countless triangles creates a sense of hierarchy. It is based on the creative elements of the geometry of the smart effect, the real realization of the artistic tension and visual performance of the rich.

\section{Application of Geometric Graphics Creative Elements in Graphic Advertisement Design}

In the advertising industry, in order to achieve the overall operational efficiency and operational advantages of the extension, we must actively use different elements and structure, that is, advertising innovation is the soul of the entire advertising, no matter what the subject of advertising, effect and application model, establish and improve a more systematic operating dimensions, to achieve advertising effectiveness, and to ensure that print ads can be integrated into more effective ideas as a support point to attract the audience. Therefore, in the print ads, the effective application of geometric graphics, creative elements and creative ideas can be integrated together to achieve the overall creative layout optimization, from the fundamental realization of the overall concrete structure of the visual, print ads to add creative geometry, The biggest advantage is vivid, can be formed on the basis of rich and colorful sense of the screen, the establishment of a more realistic form of reproduction of the object, the direct transfer of information. In the print ads in the application of geometric graphic creative elements, can improve the beauty of graphic design on the basis of, to ensure that the yuan creative design works with intuitive creativity, not only out of the concrete design of the advertising material, only the use of geometric graphic creative elements for the overall layout of the layout of the print ads, the screen neat and without losing the monotonous.

\section{Application of Geometric Graphic Elements in Graphic Advertisement Design to Improve Interest}

In the print ads in the application of geometric graphics creative elements, can enhance the texture on the basis of the picture to ensure that the design structure and design concepts can be effectively extended to ensure that the audience can virtually feel the different artistic ideas. In the process of displaying the graphic structure and the overall design model, combining the different geometric graphic elements to construct the dynamic design works. Although the print ads have no dynamic effect, but with the help of geometric creative elements to achieve dynamic experience of the upgrade, and the overall design layout and structure of the fit point is more consistent, the overall design model and design concept more real, the design level, the geometric Creative elements combined with fun design concept, the formation of an effective graphical structure and basic form, can bring more intuitive viewers entertainment.

\section{Application of Geometric Graphics in Graphic Advertisement Design to Enhance the Infectivity}

In the process of graphic advertising design, it is necessary to use the geometric graphic elements to establish a more effective image information system, so as to bring the viewer more intuitive appreciation experience, it is based on this, to integrate the different elements of information to establish a sound Complete data analysis mechanism. In addition, in the traditional print ads, mainly the use of language important visual language, can use a few simple words will be able to establish an 
effective print advertising model. In the print ads in the active application of geometric graphics, not only to pass the basic emotions, but also to enhance the overall picture of the texture and visual experience to ensure that the design structure can be intuitive transmission of emotion, thereby effectively enhance the appeal to ensure that the design structure and application, The stability of the model, but also for advertising upgrades and the overall appeal of the harmony to provide more intuitive support. Plane advertising content is rich, and the specific form is more diverse, it is the use of geometric graphics creative elements to achieve a concrete form of effective communication, the need for relevant designers to improve the design quality and design creativity, if the simple use of geometric graphics creative elements of the point of view Analysis, as the creative content, the most important thing is not only the effectiveness of the overall design model, but also to accurately convey the idea of advertising, this is the application of geometric graphics creative elements of the main reasons, more is the key to multimedia advertising design Factor [2].

In other words, in the design structure, we should actively apply different design models and control elements to ensure that the information structure and geometric elements can be combined with creative elements, which in the information transmission will be visual images and geometric creative elements combined, Improve the optimization of the overall model. Excellent print ads can arouse the sympathy of the audience, the use of geometric graphic elements of the production of print ads is the design of the more important model, but also for the advertising industry to bring new design elements and design requirements. The need for print ads to attract people's attention at the same time, improve the degree of resonance of the viewer.

\section{Path Analysis of Application of Geometric Graphics Creative Elements in Graphic Advertisement Design}

\section{The Movement of Geometric Graphics in the Design of Graphic Advertisement}

Compared with the traditional print ads, geometric graphic elements used in the design of print ads, the most important feature is the overall design structure and design effects to achieve the upgrade, point, line, face, text, color and other elements also show dynamic characteristics, can form a more practical effect and practical design requirements of the advertising model, as the basic design of graphic advertising visual elements, geometric creative elements in the advertising design can be given "life" to create a more systematic design system and elements Model to ensure that different design requirements can be met. It is based on this, geometric graphic elements of the elements, lines, face more attractive to people's attention, the construction of systematic and cannot copy the image of beauty, so that people watch advertising design works produce rich association and imagination, which for the traditional plane design model, there is a very big difference and difference. Direct use of flat geometry creative elements, as a means of expression can enhance the overall picture texture at the same time, to ensure that the relevant design model to meet the requirements of geometric graphic creative element design model to become multi-planar advertising design to open a new creative form, but also in the optimization is the visual effect on the basis of the addition of artistic charm [3]. In addition, in the design of graphic advertising, visual elements with modern design features, not only can effectively change the traditional advertising design thinking, need to use more intuitive way of expression, the geometric graphics elements as a graphic design model of the important research object, effects and the actual effect of the media. Geometric graphics creative elements of the design, spatial composition and color use are all key application model, visual expression in the traditional design aesthetics on the basis of exudes its own personality characteristics.

\section{Image Aesthetic Design of Graphic Elements in Graphic Design}

In the process of establishing the actual design structure, we should combine the relevant creative requirements and the actual operation dimension to ensure the effectiveness of the graphic design model, but also lay a solid foundation for the optimization of the visual language to ensure the design dimension and design requirements. In the graphic design model, improve the efficiency of 
communication and transmission of information, and have a variety of forms of expression. Geometric graphic elements in graphic advertising design can express different emotional requirements, and can also absorb the creative language form, which is derived from visual changes, not only the overall effect is more vivid and the design elements are more attractive, out of the rich visual effects. In addition to the traditional design model in the symmetrical balanced design requirements, rhythmic rhythm design requirements and changes in unified design requirements, the design model and the design of the design of the graphic design elements, but also to follow the graphic design language form of the law, to improve the design results, The design principle is more effective, the overall design process is more rich and comprehensive, this application model is also suitable for print advertising design, it is based on the dynamic representation of the creative elements of geometric features, can improve the overall design elements at the same time, To ensure that the design process and design requirements meet the standards for the project to lay a solid foundation for the upgrade, make full use of print space and time attributes and design architecture, can effectively integrate the creative elements of creative design, the greatest degree of people to meet the visual aesthetic needs the overall print advertising design optimization project to provide material [4].

\section{The Interactive Extension of Geometric Graphics Creative Elements in Graphic Advertisement Design}

The interaction in the design of print ads is very special, it is necessary to focus on its visual aesthetic factors, but also from a more diversified design mechanism to ensure that the design process and management of the optimization, the real point of view from the audience, the use of geometric creative elements of advertising creation, improve the overall design aesthetic and design results. In the design and creation of print ads, the design works emphasize the appreciation of the public to meet the needs of the public, the popularity, visualization and integration as a market economy under the conditions of the aesthetic trend, it is based on this, in the design work to actively use diversification factors and design concepts to ensure that the design model in line with the actual needs, but also for the optimization of geometric creative elements to provide the best creative structure. Therefore, in the process of graphic advertising design, it is necessary to effectively extend the characteristics of the wave, the effective use of interactive art of geometric graphics creative elements of the depth of processing, so as to ensure the optimization of interactive effects. The traditional sense of interaction, mainly refers to the sharing of information between the two, more emphasis on the interaction between the audience and advertising ideas, and in the application of geometric graphics creative elements, to establish a single thinking to both sides of the "activities" thinking model, to optimize the creative effect, and to ensure the overall design concept and design requirements between the stability. The interactive creativity of the print ads can improve the design effectiveness by means of the creative elements of geometric figures, and show that the participants and the advertising content reach the "thinking". It is based on this; the application of geometric graphic elements in the design of multimedia advertising not only can stimulate the enthusiasm of the viewer's interaction, but also achieve a good advertising effect [5].

\section{Conclusions}

In short, the use of geometric graphic elements in print ads can create a variety of visual graphics at the same time, the establishment of a viewer and designer interaction between, and improve the overall work of the artistic value and appreciation of the value of the need for print advertising design personnel to carry out deep excavation and thinking, for the graphic design project to provide sustainable development.

\section{References}

[1] Zhao Yuan. Visual Analysis of Creative Graphics Form in Modern Print Advertisement, Art Review, 2014,11 (12): 119-123. 
[2] Zhao Yuan. Visual Analysis of Graphic Symbolic Creative Thinking from Deconstruction to Isomorphism - Modern Print Advertisement, Inner Mongolia University, 2015.

[3] Zhao Yuan. Improved visual representation of creative graphic form language in modern print ads, Journal of Inner Mongolia University Art College, 2013,08(02):71-77.

[4] Liu Ming. Study on Graphic Language Expression of Graphic Advertisement under Semantic Role, Journal of Chifeng University (Philosophy and Social Sciences), 2015,36(10):181-182.

[5] Wang Jing, Xie Rongcai, Lv Yanmei. Talking about the Relationship between Copying and Drawing in Print Advertisement, Journal of Hengshui University ,2014,07(05):85-86,92. 